\title{
Fractalkine-Induced Endothelial Cell Migration Requires MAP Kinase Signaling
}

\author{
Michael V. Volin Nha Huynh Karolina Klosowska Rosemary D. Reyes \\ James M. Woods \\ Department of Microbiology and Immunology, Midwestern University, Chicago College of Osteopathic Medicine, \\ Downers Grove, III., USA
}

\section{Key Words}

Fractalkine $\cdot$ Chemokine $\cdot$ Angiogenesis $\cdot$ Signaling

\begin{abstract}
Background/Aims: Angiogenesis is a well-established characteristic in the rheumatoid arthritis (RA) synovial pannus. We have previously demonstrated that fractalkine ( $\mathrm{Fkn} /$ $\mathrm{CX} 3 \mathrm{CL} 1$ ) expression is significantly increased in the RA joint and that fractalkine induces angiogenesis. In this work we studied mechanisms through which Fkn functions as an angiogenic mediator. Methods: Human microvascular endothelial cells (HMVECs) and human umbilical vein endothelial cells (HUVECs) were stimulated with Fkn and analyzed by Western blotting or stained with Alexa Fluor ${ }^{\circledR} 488$ phalloidin for F-actin to characterize the time frame of cytoskeletal rearrangement. Fkn-induced HUVEC chemotaxis was performed in the presence and absence of MAP kinase inhibitors. Results: Phalloidin staining of F-actin revealed significant cytoskeletal rearrangements in HUVECs and HMVECs starting as early as 10 min after Fkn stimulation. Western blotting demonstrated that HUVEC and HMVEC stimulation with Fkn for 1-30 min resulted in phosphorylation of JNK. Fkn also induces significant phosphorylation of Erk $1 / 2$ in HUVECs over a time course ranging from 1 to $15 \mathrm{~min}$. A somewhat similar time course (5-15 min) was detected for Erk 1/2 phosphorylation in HMVECs. Inhibitors of either JNK or Erk
\end{abstract}

1/2 nearly abolish Fkn-induced HUVEC migration. Conclusions: We demonstrate that Fkn induces significant alterations in cytoskeletal structure and specifically activates the MAP kinases, JNK and Erk 1/2, both of which appear necessary for endothelial cell migration. Our results suggest that the endogenous Fkn present in the RA joint may induce angiogenesis through activation of the JNK and Erk 1/2 pathways.

Copyright $\odot 2010$ S. Karger AG, Basel

\section{Background}

Angiogenesis, the growth and proliferation of new blood vessels from preexisting ones, is important to the physiological process of wound repair, and pathophysiological processes including tumor growth and chronic inflammation as seen in rheumatoid arthritis (RA) [1-4]. Many different mediators, some proangiogenic some antiangiogenic, orchestrate the angiogenic process including chemokines such as fractalkine/CX3CL1 (Fkn) $[5,6]$.

Chemokines are 8- to $10-\mathrm{kDa}$ proteins which are subdivided into four families (C, CC, CXC, and CX3C) based on the relative position of cysteine residues [7]. Chemokines can function as leukocyte attractants and angiogenic mediators. Specifically, CXC chemokines contain-

\section{KARGER}

(C) 2010 S. Karger AG, Basel

Fax +4161306 1234

E-Mail karger@karger.ch

www.karger.com
Accessible online at: www.karger.com/pat
James M. Woods, PhD

Department of Microbiology and Immunology

Chicago College of Osteopathic Medicine, Midwestern University

555 31st Street, Downers Grove, IL 60515 (USA)

Tel. +1 630515 6173, Fax +1 630515 7245, E-Mail jwoods@ midwestern.edu 
ing the ELR motif, consisting of glutamic acid-leucinearginine preceding the CXC sequence are typically angiogenic [8]. Additionally, the CC chemokine monocyte chemotactic protein-1 (MCP-1) has been identified as an inducer of angiogenesis [9]. We have previously shown the $\mathrm{CX} 3 \mathrm{C}$ chemokine, Fkn, as a mediator of angiogenesis in RA [5]. Subsequently, others have confirmed Fkn as an angiogenic mediator [10-12].

Fkn is the only member of the CX3C chemokine family which is identified by having three amino acids between its two terminal cysteine residues [13]. Fkn is distinct from most other chemokines in that it is a large transmembrane protein containing a long negatively charged mucin-like stalk with a chemokine domain perched atop. Fkn can be cleaved resulting in the release of a soluble $95-\mathrm{kDa}$ glycoprotein. Soluble Fkn is a monomer and can function as a chemoattractant for T lymphocytes, monocytes and NK cells [14-16]. Membrane-bound Fkn mediates leukocyte adhesion to endothelial cells and leukocyte capture [17]. Functionally, we and others have previously shown Fkn to be capable of the recruitment of endothelial cells in vitro and the formation of new blood vessels in vivo [5, 10-12].

CX3CR1 is the sole receptor for Fkn and is expressed on T lymphocytes, monocytes, NK cells, and endothelial cells $[5,18]$. Chemokine receptors are known to use different cell signaling pathways involved in cell functions such as survival, proliferation, and migration. This is also true for CX3CR1 as Fkn stimulation of microglial cells has been shown to induce migration and activation of MAPK [19]. Similarly, Chinese hamster ovary cells, transfected with CX3CR1, phosphorylate MAPK and Akt in response to Fkn stimulation [20]. Recently, neuroblastoma cells expressing CX3CR1 transmigrate through endothelial monolayers and phosphorlyate extracellular signal-related kinase (Erk) 1/2 and Akt in response to soluble Fkn [21].

Expression of CX3CR1 does not by itself imply that a cell will migrate toward Fkn. For example, MonoMac6 cells and primary astrocytes both express CX3CR1 but do not migrate to Fkn $[19,22]$. Interestingly, MonoMac6 cell migration toward MCP-1 can be antagonized by Fkn [22]. The lack of migration in these cells compared to migrating cells such as human microvascular endothelial cells (HMVECs) indicates that CX3CR1 is likely coupled to different signal transduction pathways in different cell types.

We have previously shown that Fkn expression is increased in RA and that Fkn induces microvascular endothelial cell migration, in vitro tube formation, and in vivo angiogenesis $[5,23]$. The mechanism by which Fkn initi- ates these responses is not fully elucidated. In this article we use Western blot analysis to elaborate on the signaling events initiated upon Fkn stimulation of macrovascular and microvascular endothelial cells. Additionally we utilize fluorescent microscopy to identify the nature and timing of cytoskeleton changes which occur in response to Fkn stimulation of endothelial cells. Finally, we examine whether phosphorylation of MAP kinases is required for endothelial cell migration.

\section{Methods}

HMVECs and Human Umbilical Vein Endothelial Cells

HMVEC and human umbilical vein endothelial cells (HUVECs) were purchased from Cambrex (Walkersville, Md., USA) and grown in low serum media supplemented with growth factors (EGM-2MV) as recommended by the manufacturer.

\section{F-Actin Staining}

HMVECs or HUVECs were plated on gelatin-coated round glass coverslips in a 24-well tissue culture plate at either 100,000 or 50,000 cells/well in EGM-2MV. The following day cells were washed with PBS and media was replaced with serum-free basal media (EBM-2). After $1 \mathrm{~h}$ at $37^{\circ} \mathrm{C}$, media was replaced with EBM2 containing 10 or $1 \mathrm{nM}$ human Fkn (chemokine domain; R\&D Systems, Minneapolis, Minn., USA) for the times indicated (10 or $30 \mathrm{~min}, 1,2$ or $3 \mathrm{~h}$ ). Cells were fixed in $1 \mathrm{ml}$ of $3.7 \%$ formaldehyde in PBS at room temperature for $10 \mathrm{~min}$ and washed twice with PBS. Glass coverslips were removed from the plate and cells were permeabilized with acetone at $-20^{\circ} \mathrm{C}$ for $3 \mathrm{~min}$ and immediately washed twice in PBS. Coverslips were blocked in $100 \mu \mathrm{l}$ PBS $+1 \%$ BSA for 25 min. Block was replaced with Alexa Fluor ${ }^{\circledR} 488$ phalloidin (Molecular Probes, Carlsbad, Calif., USA) in PBS + 1\% BSA + DAPI $(10 \mu \mathrm{g} / \mathrm{ml})$ for $20 \mathrm{~min}$ at room temperature. Coverslips were washed twice in PBS, air dried, mounted on a microscope slide, and stored in the dark at $4^{\circ} \mathrm{C}$ until observed. Representative photographs were taken using a Nikon Eclipse E400 microscope fitted with a Spot Digital camera.

\section{Western Blotting}

HMVECs or HUVECs were cultured in EGM-2MV, washed with PBS, and then introduced to EBM-2 in the absence of serum and growth factors for $1 \mathrm{~h}$. Media was replaced with EBM-2 + 10 nM Fkn for 1, 5, 10, 15 or 30 min prior to lysing. Cells were lysed in an extraction buffer containing $20 \mathrm{~mm}$ Tris $\mathrm{pH} 7.4,137 \mathrm{mM}$ $\mathrm{NaCl}, 1 \mathrm{mM} \mathrm{Na}_{4} \mathrm{O}_{7} \mathrm{P}_{2}, 10 \mu \mathrm{M} \mathrm{NaVO}_{4}, 100 \mu \mathrm{M} \mathrm{NaF}, 1 \% \mathrm{NP}-40$ and $10 \%$ glycerol. Cell lysates were mixed 1:1 with Laemmli's sample buffer containing 20\% sodium dodecyl sulfate and boiled for $5 \mathrm{~min}$. Samples were then subjected to gel electrophoresis. Separated proteins were transferred from the gel onto a nitrocellulose membrane using a Trans-Blot ${ }^{\circledR}$ SD Electrophoretic Cell (Bio-Rad Laboratories, Hercules, Calif., USA) for $30 \mathrm{~min}$. To block nonspecific binding, membranes were incubated with $5 \%$ milk in Trisbuffered saline containing $0.1 \%$ Tween-20 (TBST) for $1 \mathrm{~h}$ at $4{ }^{\circ} \mathrm{C}$. The blots were further incubated overnight with primary antibody at $4^{\circ} \mathrm{C}$ (Cell Signaling Technology, Beverly, Mass., USA). After washing in TBST, blots were incubated with horseradish 
peroxidase-conjugated goat anti-rabbit IgG antibody (diluted 1:2,000 in TBST $+5 \%$ milk) for $1 \mathrm{~h}$ at $4^{\circ} \mathrm{C}$. ECL Plus detection reagents (Amersham, Piscataway, N.J., USA) and a Storm 860 PhosphorImager (Amersham Pharmacia Biotech, Piscataway, N.J., USA) were used in conjunction with Image Quant software (Molecular Dynamics, Sunnyvale, Calif., USA) to detect and quantitate the bands.

\section{Endothelial Cell Chemotaxis}

Briefly, HUVECs were progressively introduced to lower concentrations of serum prior to the assay by diluting EGM-2MV with EBM-2 using the following schedule: 1:2 dilution for $4 \mathrm{~h}$; $1: 4$ dilution for $4 \mathrm{~h}$; 1:8 dilution overnight (14 h); serum-free EBM-2 for $4 \mathrm{~h}$. Assays were conducted in a 48 -well microchemotaxis chamber with polycarbonate membranes containing 8 - $\mu \mathrm{m}$ pores (Neuroprobe, Cabin John, Md., USA) coated with gelatin. Stimulants were loaded into the bottom half of the chamber, while the top contained 40,000 HUVECs/well. Where indicated, MEK [the activator of Erk 1/2] was inhibited using U0126 (30 $\mu \mathrm{M})$ and Jun$\mathrm{N}$-terminal kinase (JNK) was inhibited using SP600125 (30 $\mu \mathrm{M})$, while the vehicle was $0.1 \%$ DMSO. Migration proceeded for $4 \mathrm{~h}$ at $37^{\circ} \mathrm{C}$, after which the nonmigrated cells were removed with a cotton swab, cells were fixed to the membrane, and stained with DiffQuick (Dade-Behring, Deerfield, Ill., USA). Each sample was assayed at least in quadruplicate, and migrated cells were counted in triplicate high-powered fields selected randomly.

\section{Results}

Fkn stimulation of HUVECs induces F-actin reorganization. Actin reorganization, a prerequisite for endothelial cell migration and angiogenesis, was determined using Alexa Fluor 488 phalloidin, a fluorescent stain for filamentous actin. HUVEC stimulation with Fkn, at either 1 or $10 \mathrm{nM}$, concentrations which are known to induce endothelial cell migration [10], can cause reorganization of actin filaments (fig. 1a-f). Subconfluent nonstimulated HUVECs exhibited diffuse actin arrangements, which often could be barely seen, as shown in figure 1a. Within 10- to 30-min exposure of HUVECs to Fkn, Alexa Fluor 488 phalloidin staining of actin filaments intensifies and noticeable organization of thin actin cables can be seen stretching the length of the cell (fig. 1b, c). After 1 h of Fkn stimulation of HUVECs there is prominent staining of F-actin (fig. 1d). The most obvious differences in F-actin labeling were observed after 2 $\mathrm{h}$ stimulation with Fkn, where intense actin filament staining is seen stretching from one end to the other end of the cell (fig. 1e). Figure 1 shows representative photographs of HUVECs stimulated with $1 \mathrm{nM}$ Fkn $(\mathrm{n}=4)$. Stimulation with $10 \mathrm{nM}$ Fkn resulted in nearly identical observations ( $\mathrm{n}=4$, data not shown).

Fractalkine Signaling in Endothelial Cells
Fkn stimulation of HMVECs induces F-actin reorganization. Next, Fkn-induced actin reorganization was studied in microvascular endothelial cells, HMVECs. We have previously shown that stimulation with Fkn at concentrations of either 1 or $10 \mathrm{nM}$ can induce HMVEC migration [5]. In figure 2 we show that Fkn at $1 \mathrm{nM}$ can cause reorganization of actin filaments $(n=2)$. Stimulation with $10 \mathrm{nM}$ Fkn resulted in nearly identical observations ( $\mathrm{n}=2$, data not shown). Subconfluent nonstimulated HMVECs exhibited diffuse actin arrangements, which often could be barely seen, as shown in figure 2 a. However, just as we observed in HUVECs, HMVECs stimulated with Fkn for as little as 10 min began to have increased F-actin staining with organization of thin actin cables that can be seen stretching the length of the cell (fig. 2b). F-actin staining continues to increase in intensity by $2 \mathrm{~h}$ of Fkn stimulation (fig. 2e). Figure 2 shows representative photographs of 2 independent HMVECs stimulations with $1 \mathrm{nM}$ Fkn.

Fkn stimulation of endothelial cells does not induce signaling through Akt. In other cell types including RA synovial fibroblasts, Fkn induces signals through CX3CR1 linked to a variety of pathways including the survival pathways involving Akt [19, 24-26]. We performed Western blot analysis on Fkn-stimulated endothelial cell lysates using phosphospecific antibodies for either Thr 308 or Ser 473 in order to study changes in Akt phosphorylation. These studies revealed that HMVECs and HUVECs have a basal level of Akt phosphorylation at both Thr 308 and Ser 473 without Fkn stimulation. Fkn stimulation (10 $\mathrm{nM}$ ) of HMVECs or HUVECs for up to 30 min did not dramatically alter the basal levels of Akt phosphorylation at either Thr 308 or Ser 473 (fig. 3).

Fkn stimulation of endothelial cells induces signaling through MAPK family members. Fkn stimulation of other cell types is known to activate JNK $[19,24,25]$. To determine whether Fkn stimulation of HMVECs and HUVECs activates JNK, we stimulated cells with $10 \mathrm{nM} \mathrm{Fkn}$ for various time periods and used Western blotting with a phosphospecific antibody on cell lysates. Figure $4 \mathrm{a}$ demonstrates that some basal phosphorylation of JNK could be observed in nonstimulated HMVECs (0 min), but a prominent enhancement was detected starting as early as $1 \mathrm{~min}$ and peaking after $5 \mathrm{~min}$ of stimulation. Enhancement of JNK phosphorylation by Fkn was detected in cell lysates from three separate stimulations of HMVECs. A graphical representation of arbitrary band intensity of phospho-JNK normalized to the total amount of JNK detected is also shown. Figure $4 \mathrm{~b}$ demonstrates minimal phosphorylation of JNK could be observed in 

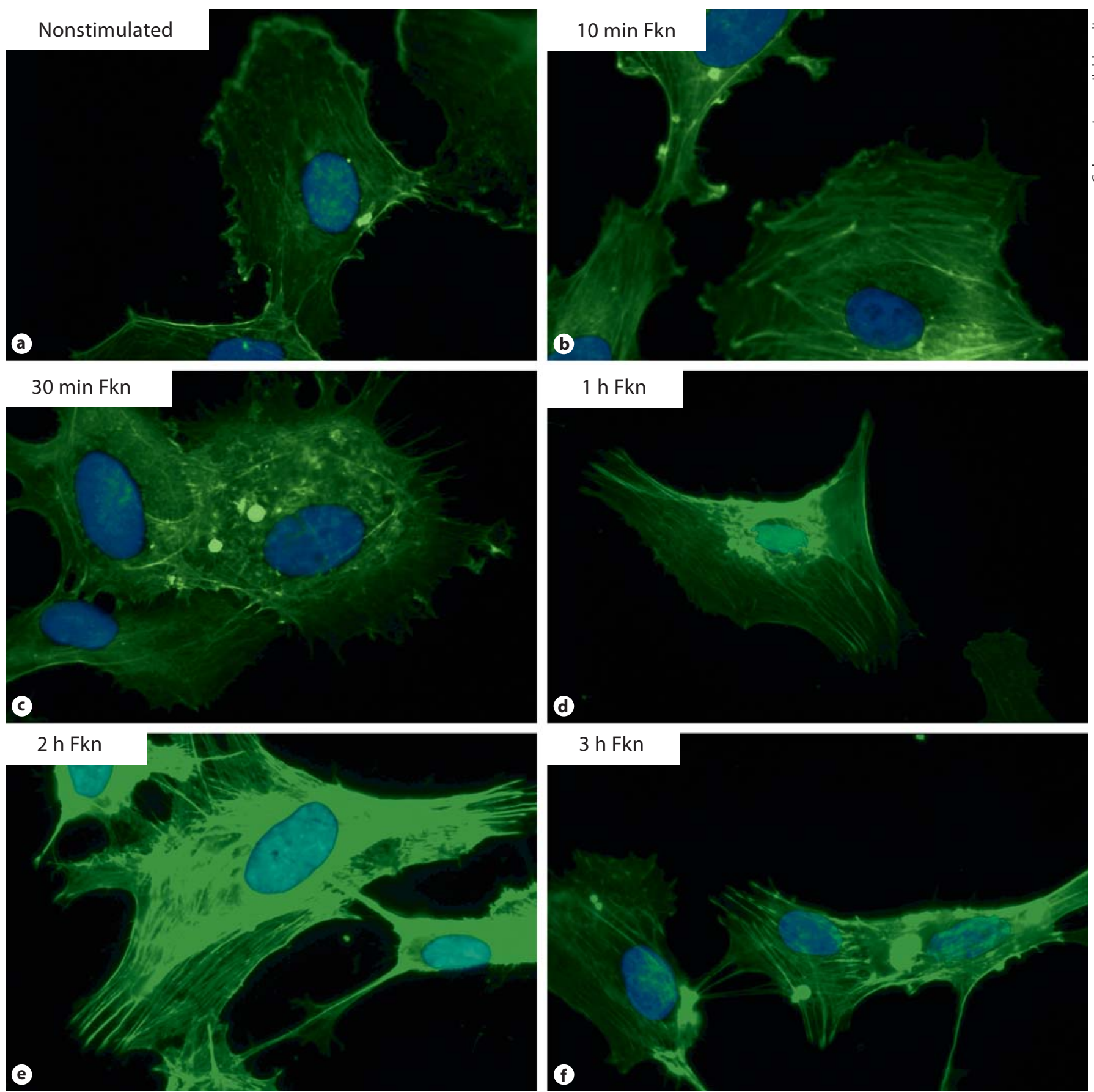

\section{$3 \mathrm{~h} \mathrm{Fkn}$}

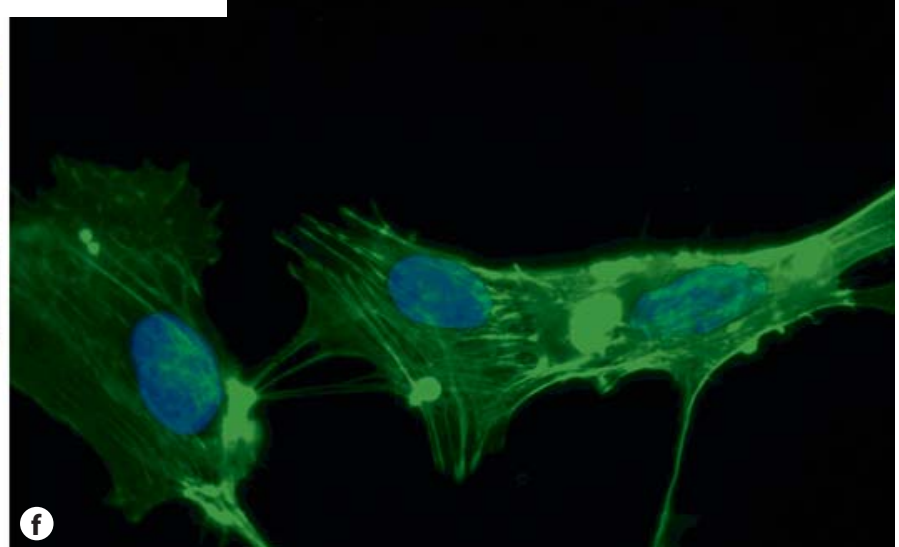

Fig. 1. a Background staining of F-actin in nonstimulated HUVECs. Cells stimulated with $1 \mathrm{~nm}$ Fkn and stained for F-actin after $10 \mathrm{~min}(\mathbf{b}), 30 \mathrm{~min}(\mathbf{c}), 1 \mathrm{~h} \mathrm{(d),} 2 \mathrm{~h} \mathrm{(e),} \mathrm{and} 3 \mathrm{~h}(\mathbf{f})$ are shown for comparison. Stimulation with $10 \mathrm{nM}$ Fkn resulted in a nearly identical time course of F-actin reorganization (data not shown). Original magnification $\times 1,000$. 

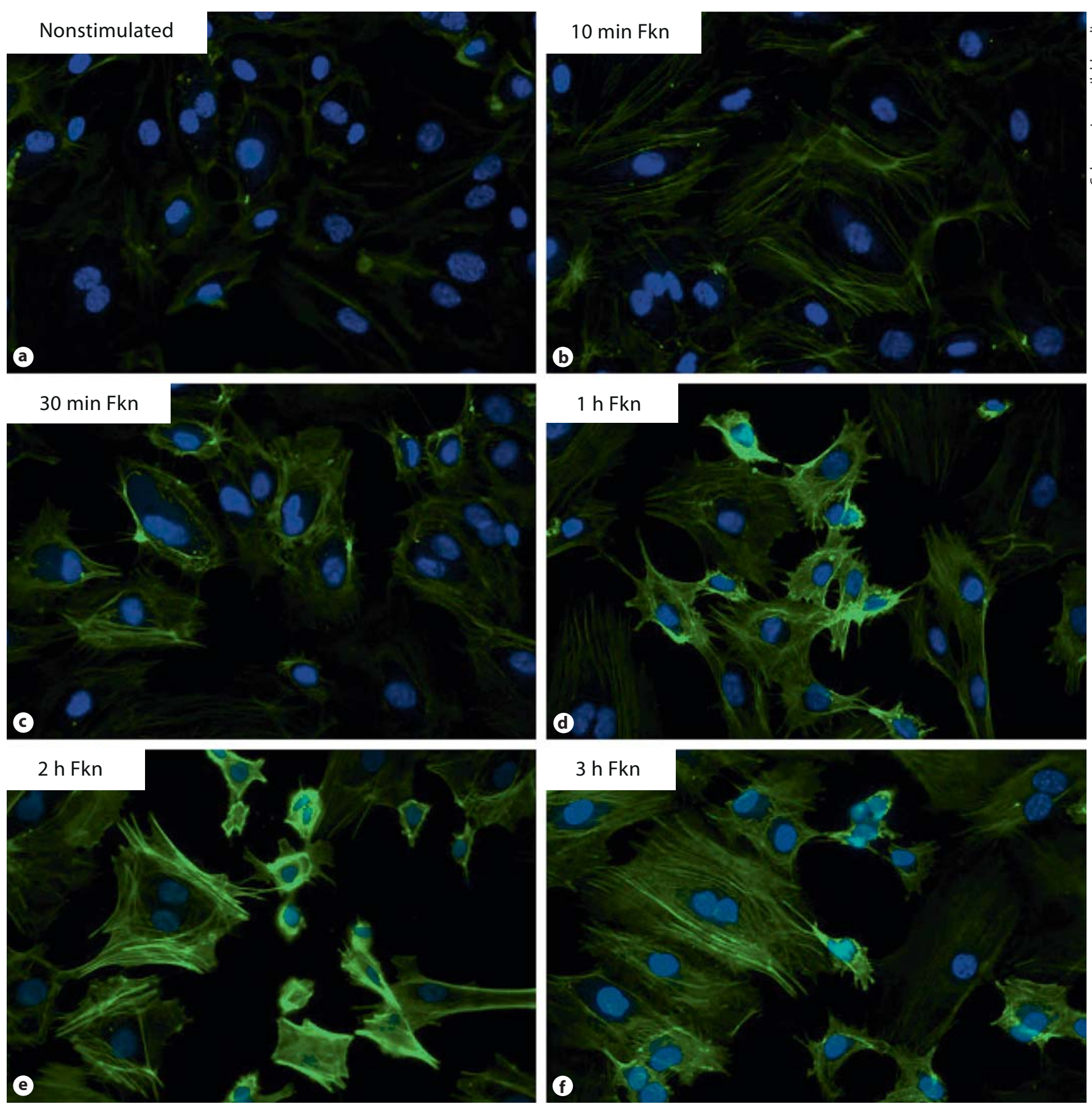

Fig. 2. a Background staining of F-actin in nonstimulated HMVECs. Cells stimulated with $1 \mathrm{nM}$ Fkn and stained for F-actin after $10 \mathrm{~min}(\mathbf{b}), 30 \mathrm{~min}(\mathbf{c}), 1 \mathrm{~h} \mathrm{(d),} 2 \mathrm{~h} \mathrm{(e),} \mathrm{and} 3 \mathrm{~h}(\mathbf{f})$ are shown for comparison. Stimulation with $10 \mathrm{~nm}$ Fkn resulted in a nearly identical time course of F-actin reorganization (data not shown). Original magnification $\times 400$. 


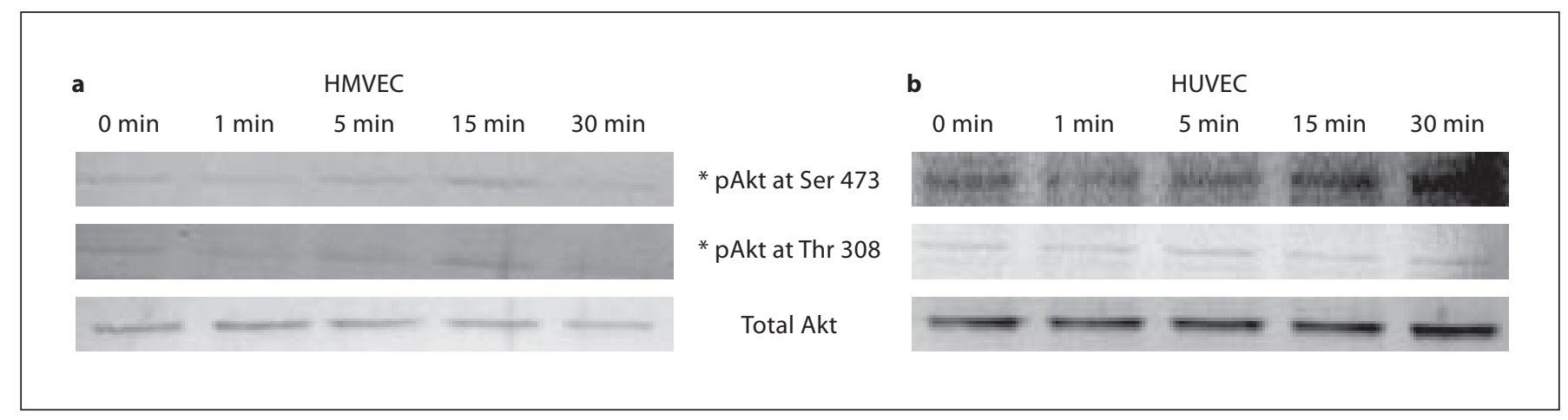

Fig. 3. HMVECs (a) or HUVECs (b) had serum and growth factors removed for $1 \mathrm{~h}$ and were then stimulated with basal media containing $10 \mathrm{nM}$ Fkn in basal media for the times indicated. Cell lysates were generated and $20 \mu \mathrm{g}$ protein/lane was separated by PAGE and then blotted using standard Western blotting techniques. Phosphorylated Akt was compared with total Akt to assure equal loading. Blots are representative of three independent experiments.

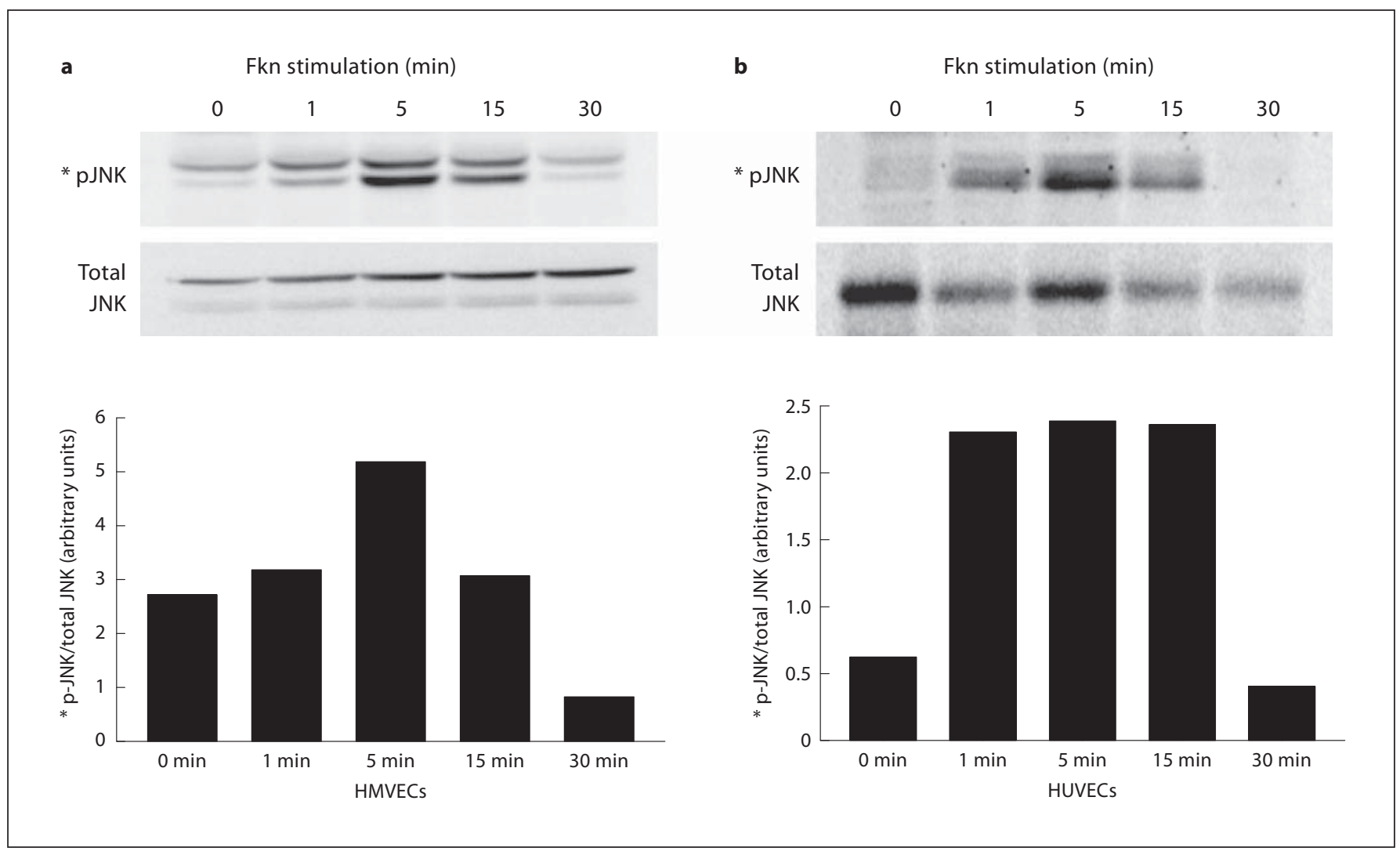

Fig. 4. Fkn (10 nM) was added to HMVECs (a) or HUVECs (b) for the times indicated and cell lysates were generated. Protein $(20 \mu \mathrm{g} / \mathrm{lane})$ was separated using PAGE and then blotted using standard Western techniques. Phosphorylated JNK was compared with total JNK or actin to assure equal loading. Bars represent normalized band intensity in arbitrary units. Blots are representative of three independent experiments. 


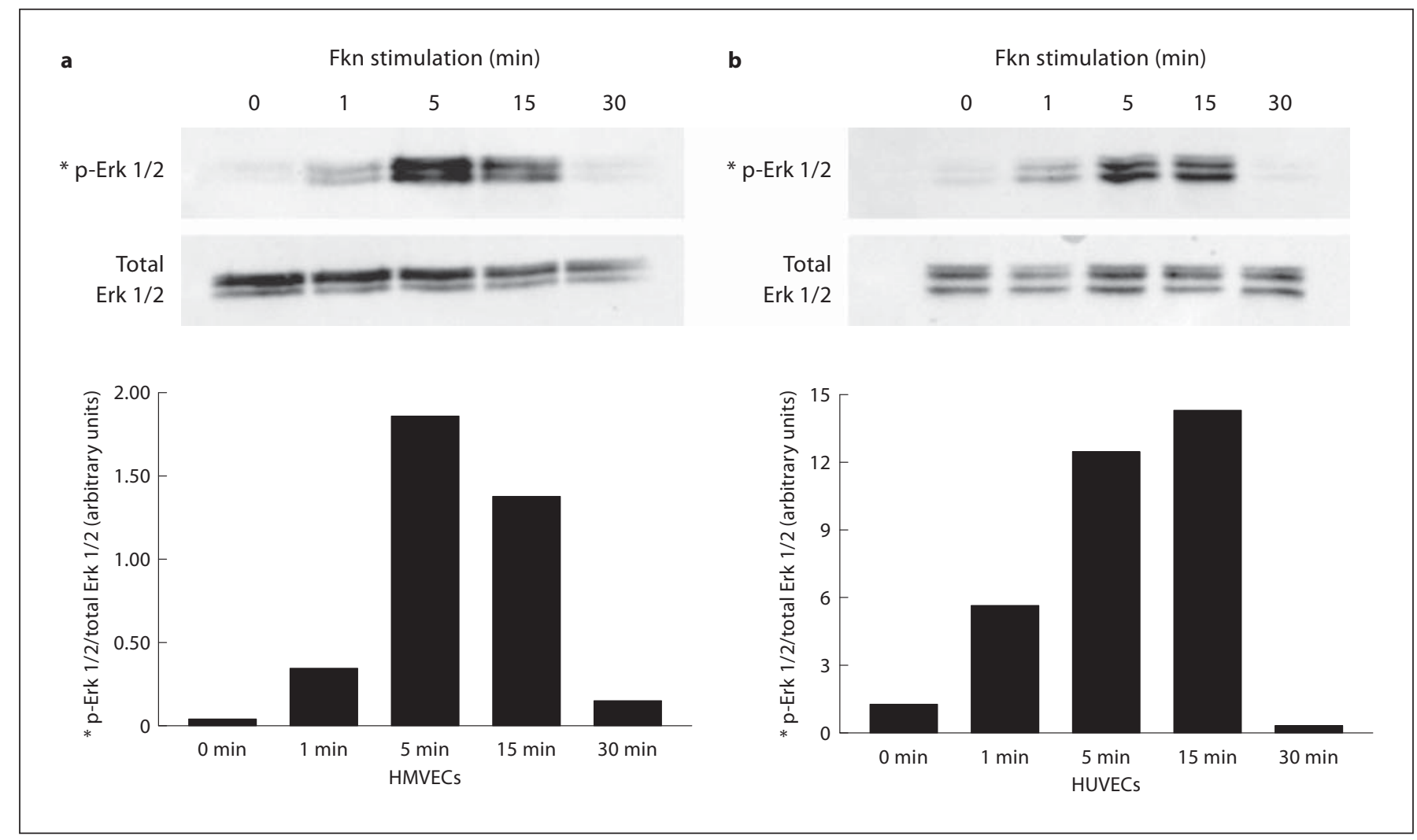

Fig. 5. Fkn (10 nM) was added to HMVECs (a) or HUVECs (b) for the times indicated and cell lysates were generated. Protein $(20 \mu \mathrm{g} / \mathrm{lane})$ was separated using PAGE and then blotted using standard Western techniques. Phosphorylated Erk 1/2 was compared with total Erk 1/2 to assure equal loading. Bars represent normalized band intensity in arbitrary units. Blots are representative of three independent experiments.

nonstimulated HUVECs (0 min), but a prominent enhancement was detected starting as early as $1 \mathrm{~min}$ and peaking after 5 min of stimulation. Enhancement of JNK phosphorylation by Fkn was detected in cell lysates from three separate stimulations of HUVECs. A graphical representation of arbitrary band intensity of phospho-JNK normalized to the total amount of JNK detected is also shown.

Cell lysates were also examined for Fkn-induced phosphorylation of Erk 1/2 which is known to be activated in other cell types $[19,24,25]$. To determine whether Fkn stimulation of HMVECs and HUVECs activates Erk 1/2, we stimulated cells with $10 \mathrm{nM}$ Fkn for various time periods and used Western blotting with a phosphospecific antibody on cell lysates. Figure $5 \mathrm{a}$ demonstrates that minimal phosphorylation of Erk 1/2 could be observed in nonstimulated HMVECs (0 min), but a prominent enhancement was detected starting as early as $1 \mathrm{~min}$ and peaking after $5 \mathrm{~min}$ of stimulation. Enhancement of Erk
1/2 phosphorylation by Fkn was detected in cell lysates from three separate Fkn stimulations of HMVECs. A graphical representation of arbitrary band intensity of phospho-Erk 1/2 normalized to the total amount of Erk $1 / 2$ detected is also shown. Figure $5 \mathrm{~b}$ demonstrates that minimal phosphorylation of Erk 1/2 could be observed in nonstimulated HUVECs (0 min), but a prominent enhancement was detected starting as early as $1 \mathrm{~min}$ and peaking after $15 \mathrm{~min}$ of stimulation. Enhancement of Erk 1/2 phosphorylation by Fkn was detected in cell lysates from three separate Fkn stimulations of HUVECs. A graphical representation of arbitrary band intensity of phospho-Erk 1/2 normalized to the total amount of Erk $1 / 2$ detected is also shown.

Cell migration is a critical component of angiogenesis which requires reorganization of actin. Since Fkn stimulates angiogenesis and MAP kinase phosphorylation, we next determined whether inhibition of the MAP kinases JNK and Erk would inhibit HUVEC migration. Data 


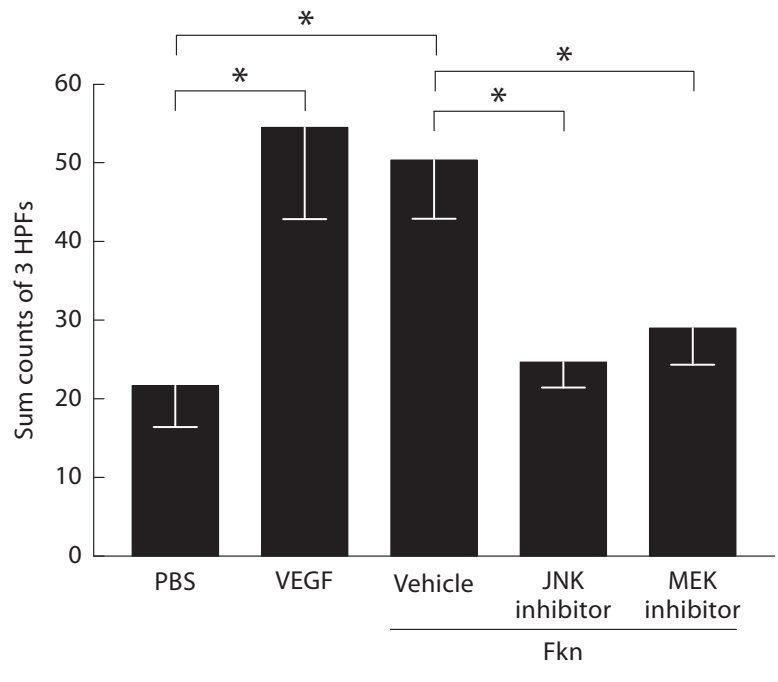

Fig. 6. HUVEC migration induced by VEGF (100 nM) is shown as a positive control compared with PBS demonstrating background migration. Fkn (100 nM) was used to induce HUVEC migration in the presence and absence of a JK (SP600125; $30 \mu \mathrm{M})$ and MEK (U0126; $30 \mu \mathrm{M})$ inhibitor. DMSO (0.1\%) was included as a vehicle control. Bars show the mean and standard error of the mean of counts summed from 3 high-powered microscope fields (HPFs) from replicate wells. The graph represents 4 separate experiments, where statistical analysis was performed using one-way analysis of variance and a post-hoc Newman-Keuls test $\left({ }^{*} \mathrm{p}<0.01 ; \mathrm{n}=4\right)$.

shown in figure 6 demonstrate that significant HUVEC migration can be induced by Fkn, but that same migration can be significantly decreased by inhibiting either JNK or Erk 1/2 in these cells. Inhibition of Erk 1/2 was accomplished using U0126, which is an inhibitor of MEK $1 / 2$, the kinase that activates Erk 1/2. Thus, Fkn-induced endothelial cell migration appears dependent on signaling through both JNK and Erk 1/2.

\section{Discussion}

In previous studies we and others have shown that the angiogenic properties of Fkn are similar in potency to other angiogenic chemokines such as IL-8 and ENA-78 $[5,27]$. Specifically, $1 \mathrm{~nm}$ Fkn can induce a doubling of HMVEC chemotaxis as well as significant migration at lower concentrations. Moreover, Fkn induces angiogenesis in vivo, while Fkn found in RA synovial fluid and synovial tissue homogenates also contribute to angiogen- esis [5]. The angiogenic process is multifaceted involving endothelial cell migration, proliferation, elongation, orientation, and differentiation ultimately resulting in basement membrane reestablishment, lumen formation, and anastomosis with other vessels. Cell migration can be divided up into four distinct steps: lamellipodium extension, formation of new adhesions, contraction of the cell body, and tail detachment [28]. The actin cytoskeleton is intricately involved in both the extension of lamellipodium and the contraction of cell bodies.

While Fkn was known to induce migration of endothelial cells, cytoskeletal changes that result from Fkn stimulation have not been documented. Actin reorganization is known to be a prerequisite for migration of many different cell types [29]. In this study, we describe changes in actin staining intensity and actin cytoplasmic location in HMVECs and HUVECs upon Fkn stimulation. The influence of Fkn stimulation on the actin network was analyzed by fluorescence staining. Fkn stimulation for as little as $10 \mathrm{~min}$ results in increased actin staining showing more pronounced actin fibers leading to the cell edges. By $2 \mathrm{~h}$ of Fkn stimulation there is pronounced staining of actin fibers throughout the cytoplasm in both HUVECs and HMVECs. A hallmark of endothelial cell migration is the formation of actin bundles through the cell body in the direction of cell movement [30]. Thus, in the same time frame in which we previously showed Fkn to induce endothelial cell migration we now show actin cytoskeletal rearrangements elaborating upon the close relationship between the cytoskeleton and cell mobility.

It is understood that soluble Fkn initiates endothelial cell migration and angiogenesis by interacting with its receptor CX3CR1 [5, 10-12]. Chemokine receptors are G protein-coupled serpentine receptors and are known to utilize different signaling mechanisms for different functions [31]. In other cell types Fkn has been shown to signal through the MAPK family and through Akt downstream of $G$ protein activation $[19,24,25]$. Here we show phosphorylation of MAPK proteins Erk 1/2 and JNK shortly after Fkn stimulation of both venous and microvascular endothelial cells. This is in agreement with a report by Lee et al. [10] showing HUVEC chemotaxis and tube formation to be dependent upon Erk 1/2 phosphorylation. Additionally, we were able to eliminate Fkn-induced migration of venous endothelial cells by inhibiting either Erk $1 / 2$ or JNK using specific inhibitors.

Other angiogenic mediators including tumor necrosis factor- $\alpha$ and vascular endothelial growth factor (VEGF) also induce endothelial cell signaling through the MAP kinase family of molecules. Tumor necrosis factor- $\alpha$ 
stimulation of either HUVECs or HMVECs has been shown to activate Erk and JNK [32,33]. It is also known that endothelial cells isolated from different parts of the circulatory system utilize different signaling molecules when stimulated with the same angiogenic mediator. Specifically, it has been shown that VEGF stimulation of microvascular endothelial cells results in Ras-dependent Erk phosphorylation while this is not the case in HUVECs [34]. The signaling mechanisms involved in specific cellular functions also vary by angiogenic mediator and cell type. HUVECs stimulated with VEGF migrate independently of Erk phosphorylation while our work shows that Fkn-stimulated HUVEC migration can be reduced by an inhibitor of Erk phosphorylation [35].

One way in which MAPK activation may be involved in cell migration is through the myosin light chain kinase. Activation of the MAPKs is known to stimulate myosin light chain kinase ultimately leading to actomyosin contractility and cell body contraction, the third step necessary for cell migration [36]. Additionally, Erk is known to be a regulator of protease activity that is involved in detachment of focal adhesion components necessary for tail detachment [37]. Recently, Ryu et al. [11] showed that in human aortic endothelial cells Fkn stimulation resulted in upregulation of Hif-1alpha which in turn induced VEGF-A expression through an Erk 1/2 pathway. This result is in conflict with a previous report showing that VEGF was not necessary for Fkn-induced angiogenesis [10].

Fkn stimulation of HUVECs or HMVECs did not result in phosphorylation of Akt at either Ser 473 or Thr 308 through $30 \mathrm{~min}$ of stimulation suggesting that this sur- vival pathway is not activated by Fkn. We chose time points of 30 min or less based on previous studies of various cell types stimulated with Fkn which showed Akt phosphorylation before or at $30 \mathrm{~min}[24,26,38,39]$. Additionally, Akt activation in endothelial cells stimulated with cytokines other than Fkn showed phosphorylation of Akt before $30 \mathrm{~min}$ [40]. However, a recent report by Lee et al. [10] showed that Fkn-stimulated HUVECs did phosphorylate Akt, but only after $45 \mathrm{~min}$. Thus, it may take a longer stimulation with Fkn to stimulate Akt in HMVECs.

\section{Conclusion}

Fkn is an angiogenic chemokine found in RA joints that is known to induce endothelial cell migration and tube formation. We show that Fkn induces both microvascular and macrovascular endothelial cells to remodel their cytoskeleton within minutes of exposure. At this same time, Fkn-stimulated endothelial cells phosphorylate MAP kinases JNK and Erk 1/2, but not Akt. Activation of both JNK and Erk 1/2 appear critical to endothelial cell migration and therefore, in turn, are likely critical to Fkn-induced angiogenesis.

\section{Acknowledgments}

This work was funded by an Arthritis Foundation Arthritis Investigator Award (to J.M.W.) and NIH grant R15AR050985 (to J.M.W.).

\section{References}

1 Rudolph EH, Woods JM: Chemokine expression and regulation of angiogenesis in rheumatoid arthritis. Curr Pharm Des 2005;11: 613-631.

-2 Szekanecz Z, Gaspar L, Koch AE: Angiogenesis in rheumatoid arthritis. Front Biosci 2005; 10:1739-1753.

- 3 Folkman J: Role of angiogenesis in tumor growth and metastasis. Semin Oncol 2002; 29:15-18.

4 Li J, Zhang YP, Kirsner RS: Angiogenesis in wound repair: angiogenic growth factors and the extracellular matrix. Microsc Res Tech 2003;60:107-114.

5 Volin MV, Woods JM, Amin MA, Connors MA, Harlow LA, Koch AE: Fractalkine: a novel angiogenic chemokine in rheumatoid arthritis. Am J Pathol 2001;159:1521-1530.
-6 Sun XT, Zhang MY, Shu C, et al: Differential gene expression during capillary morphogenesis in a microcarrier-based three-dimensional in vitro model of angiogenesis with focus on chemokines and chemokine receptors. World J Gastroenterol 2005;11: 2283-2290.

7 Baggiolini M: Chemokines and leukocyte traffic. Nature 1998;392:565-568.

-8 Strieter RM, Polverini PJ, Kunkel SL, et al: The functional role of the ELR motif in CXC chemokine-mediated angiogenesis. J Biol Chem 1995;270:27348-27357.

-9 Goede V, Brogelli L, Ziche M, Augustin HG: Induction of inflammatory angiogenesis by monocyte chemoattractant protein-1. Int J Cancer 1999;82:765-770.
10 Lee SJ, Namkoong S, Kim YM, et al: Fractalkine stimulates angiogenesis by activating the Raf-1/MEK/ERK- and PI3K/Akt/eNOSdependent signal pathways. Am J Physiol Heart Circ Physiol 2006;291:H2836-H2846.

-11 Ryu J, Lee CW, Hong KH, et al: Activation of fractalkine/CX3CR1 by vascular endothelial cells induces angiogenesis through VEGF$\mathrm{A} / \mathrm{KDR}$ and reverses hindlimb ischaemia. Cardiovasc Res 2008;78:333-340.

12 You JJ, Yang CH, Huang JS, Chen MS, Yang $\mathrm{CM}$ : Fractalkine, a CX3C chemokine, as a mediator of ocular angiogenesis. Invest Ophthalmol Vis Sci 2007;48:5290-5298.

13 Bazan JF, Bacon KB, Hardiman G, et al: A new class of membrane-bound chemokine with a CX3C motif. Nature 1997;385:640644. 
14 Schall T: Fractalkine - a strange attractor in the chemokine landscape. Immunol Today 1997; 18:147.

15 Al-Aoukaty A, Rolstad B, Giaid A, Maghazachi AA: MIP-3alpha, MIP-3beta and fractalkine induce the locomotion and the mobilization of intracellular calcium, and activate the heterotrimeric $\mathrm{G}$ proteins in human natural killer cells. Immunology 1998;95:618624.

16 Ancuta P, Moses A, Gabuzda D: Transendothelial migration of CD16+ monocytes in response to fractalkine under constitutive and inflammatory conditions. Immunobiology 2004;209:11-20.

17 Fong AM, Robinson LA, Steeber DA, et al: Fractalkine and CX3CR1 mediate a novel mechanism of leukocyte capture, firm adhesion, and activation under physiologic flow. J Exp Med 1998;188:1413-1419.

18 Zhang Q, Shimoya K, Temma K, et al: Expression of fractalkine in the Fallopian tube and of CX3CR1 in sperm. Hum Reprod 2004;19:409-414.

19 Maciejewski-Lenoir D, Chen S, Feng L, Maki $\mathrm{R}, \mathrm{Bacon} \mathrm{KB}$ : Characterization of fractalkine in rat brain cells: migratory and activation signals for CX3CR-1-expressing microglia. J Immunol 1999;163:1628-1635.

-20 Kansra V, Groves C, Gutierrez-Ramos JC, Polakiewicz RD: Phosphatidylinositol 3-kinase-dependent extracellular calcium influx is essential for CX(3)CR1-mediated activation of the mitogen-activated protein kinase cascade. J Biol Chem 2001;276:31831-31838.

21 Nevo I, Sagi-Assif O, Meshel T, et al: The involvement of the fractalkine receptor in the transmigration of neuroblastoma cells through bone-marrow endothelial cells. Cancer Lett 2009;273:127-139.

22 Vitale S, Schmid-Alliana A, Breuil V, et al: Soluble fractalkine prevents monocyte chemoattractant protein-1-induced monocyte migration via inhibition of stress-activated protein kinase $2 / \mathrm{p} 38$ and matrix metalloproteinase activities. J Immunol 2004;172:585592 .
-23 Ruth JH, Volin MV, Haines GK 3rd, et al: Fractalkine, a novel chemokine in rheumatoid arthritis and in rat adjuvant-induced arthritis. Arthritis Rheum 2001;44:15681581.

24 Shulby SA, Dolloff NG, Stearns ME, Meucci O, Fatatis A: CX3CR1-fractalkine expression regulates cellular mechanisms involved in adhesion, migration, and survival of human prostate cancer cells. Cancer Res 2004; 64:4693-4698.

25 Cambien B, Pomeranz M, Schmid-Antomarchi H, et al: Signal transduction pathways involved in soluble fractalkine-induced monocytic cell adhesion. Blood 2001;97: 2031-2037.

26 Volin MV, Huynh N, Klosowska K, Chong KK, Woods JM: Fractalkine is a novel chemoattractant for rheumatoid arthritis fibroblast-like synoviocyte signaling through MAP kinases and Akt. Arthritis Rheum 2007;56:2512-2522.

27 Koch AE, Polverini PJ, Kunkel SL, et al: Interleukin- 8 as a macrophage-derived mediator of angiogenesis. Science 1992;258:17981801.

28 Ridley AJ: Rho GTPases and cell migration. J Cell Sci 2001;114:2713-2722.

-29 Tenscher K, Metzner B, Hofmann C, Schopf E, Norgauer J: The monocyte chemotactic protein-4 induces oxygen radical production, actin reorganization, and CD11b upregulation via a pertussis toxin-sensitive Gprotein in human eosinophils. Biochem Biophys Res Commun 1997;240:32-35.

30 Bijman MN, van Nieuw Amerongen GP Laurens N, van Hinsbergh VW, Boven E: Microtubule-targeting agents inhibit angiogenesis at subtoxic concentrations, a process associated with inhibition of Rac1 and Cdc42 activity and changes in the endothelial cytoskeleton. Mol Cancer Ther 2006;5:23482357.

-31 Viola A, Contento RL, Molon B: T cells and their partners: the chemokine dating agency. Trends Immunol 2006;27:421-427.

>32 Kroon ME, Koolwijk P, van der Vecht B, van Hinsbergh VW: Hypoxia in combination with FGF-2 induces tube formation by human microvascular endothelial cells in a fibrin matrix: involvement of at least two signal transduction pathways. J Cell Sci 2001; 114:825-833.
33 Yoshizumi M, Fujita Y, Izawa Y, et al: Ebselen inhibits tumor necrosis factor-alpha-induced c-Jun N-terminal kinase activation and adhesion molecule expression in endothelial cells. Exp Cell Res 2004;292:1-10.

34 Yashima R, Abe M, Tanaka K, et al: Heterogeneity of the signal transduction pathways for VEGF-induced MAPKs activation in human vascular endothelial cells. J Cell Physiol 2001;188:201-210.

>35 Rousseau S, Houle F, Landry J, Huot J: p38 MAP kinase activation by vascular endothelial growth factor mediates actin reorganization and cell migration in human endothelial cells. Oncogene 1997;15:2169-2177.

-36 Hansen SH, Zegers MM, Woodrow M, et al: Induced expression of Rnd3 is associated with transformation of polarized epithelial cells by the Raf-MEK-extracellular signalregulated kinase pathway. Mol Cell Biol 2000;20:9364-9375.

>37 Glading A, Chang P, Lauffenburger DA, Wells A: Epidermal growth factor receptor activation of calpain is required for fibroblast motility and occurs via an ERK/MAP kinase signaling pathway. J Biol Chem 2000; 275:2390-2398.

38 Lee BC, Lee TH, Avraham S, Avraham HK: Involvement of the chemokine receptor CXCR4 and its ligand stromal cell-derived factor lalpha in breast cancer cell migration through human brain microvascular endothelial cells. Mol Cancer Res 2004;2:327338.

$\checkmark 39$ Davis CN, Harrison JK: Proline 326 in the C terminus of murine CX3CR1 prevents Gprotein and phosphatidylinositol 3-kinasedependent stimulation of Akt and extracellular signal-regulated kinase in Chinese hamster ovary cells. J Pharmacol Exp Ther 2006;316:356-363.

40 Murao K, Ohyama T, Imachi H, et al: TNFalpha stimulation of MCP-1 expression is mediated by the Akt/PKB signal transduction pathway in vascular endothelial cells. Biochem Biophys Res Commun 2000;276: 791-796. 